\title{
Reporte estadístico de pacientes pediátricos atendidos en la clínica de la Universidad Tecnológica del Suroeste de Guanajuato
}

\section{Pediatric patients' statistics report attended in the clinic of Universidad Tecnologica del Suroeste de Guanajuato}

\author{
ALVAREZ-AVILA, Alejandra $\uparrow^{*} \&$ SASIA-ZAYAS, Karen \\ Universidad Tecnológica del Suroeste de Guanajuato- Departamento de Terapia Física. arretera Valle de Santiago - \\ Huamimaro Km. 1.2, 20 de Noviembre, 38400 Valle de Santiago, Gto.
}

ID 1 ${ }^{\text {er }}$ Autor: Alejandra, Alvarez-Avila / ORC ID: 0000-0001-9567-0194

ID $1^{\text {er }}$ Coautor: Karen, Sasia-Zayas / ORC ID: 0000-0001-7243-0753

DOI: $10.35429 / J O H S .2019 .20 .6 .1 .7$

Recibido Julio 5, 2019; Aceptado Septiembre 17, 2019

Resumen

El desarrollo psicomotor (DPM) es un proceso mediante el cual el niño adquiere habilidades en distintas áreas que le permiten una independencia y adaptación progresiva con el medio en el que se rodea y desenvuelve. Es fundamental la valoración adecuada del desarrollo psicomotor desde el periodo neonatal para obtener oportunas derivaciones de los casos con sospecha de enfermedad y detección de signos anómalos que desencadenen un retraso cronológico significativo en el niño. Objetivo. Conocer los datos estadísticos que evidencian las áreas del desarrollo psicomotor mayormente afectadas en pacientes pediátricos. Metodología. Estudio observacional, descriptivo, de corte transversal. Se estudiaron 51 pacientes, evaluados con historia clínica y Gesell. El período de investigación fue de Enero 2018 a Enero 2019 Contribución. Crear conciencia en la importancia de la valoración del desarrollo psicomotor en neonatos y pediátricos que presenten alguna alteración en las distintas áreas del desarrollo.

Desarrollo psicomotor, Retraso cronológico, Persistencia de patrones

\begin{abstract}
The psychomotor development is a process through which, children acquire abilities in different areas that allow them to have progressive independence and adaptation within their development environment. An adequate evaluation of the psychomotor development since neonatal period is fundamental to obtain an appropriate derivation of suspected illness cases and detection of anomalous signs that trigger a significant chronological delay in children. Objective. To know statistic numbers making evident the psychomotor develop areas majorly affected within pediatric patients. Methodology. Observational, descriptive, cross-sectional study. 51 patients were studied, in the 5 behaviors, evaluated with clinical history and Gesell. The study was realized to the January 2018 a January 2019. Contribution. Create conscience about the importance of psychomotor development evaluation in neonatal children and pediatric whether they show or not, any alteration in the different development areas.
\end{abstract}

Psychomotor development, Chronological delay, Pattern persistence

Citación: ALVAREZ-AVILA, Alejandra \& SASIA-ZAYAS, Karen. Reporte estadístico de pacientes pediátricos atendidos en la clínica de la Universidad Tecnológica del Suroeste de Guanajuato. Revista de Ciencias de la Salud. 2019. 6-20: 1-7.

$\dagger$ Investigador contribuyendo como primer Autor 


\section{Introducción}

\section{Desarrollo psicomotor}

El término desarrollo psicomotor (DPM) se atribuye al neuropsiquiatra alemán Carl Wernicke (1848-1905), quien lo utilizó para referirse al fenómeno evolutivo de adquisición continua y progresiva de habilidades a lo largo de la infancia. (Vericat,2012). El desarrollo es un proceso complejo, sus diferentes aspectos (motor, afectivo, cognitivo y social) se hallan interrelacionados e influyen mutuamente, condicionados por el medio en el que tiene lugar el desarrollo infantil. (Schonhaut, 2008).

No se puede considerar desarrollo psicomotor como algo que simplemente le va aconteciendo al niño, sino que, por el contrario, es algo que el niño va a ir produciendo a través de su deseo de actuar sobre el entorno, de ser cada vez más competente. Por eso, la meta del desarrollo psicomotor es conseguir el dominio y control del propio cuerpo, hasta ser capaz de sacar de él todas las posibilidades de acción y expresión que a cada uno le sean posibles (Martínez, 2014)

\section{Alteraciones o problemas del desarrollo}

Cuando se hace referencia al desarrollo psicomotor normal se habla de un proceso que permite al niño adquirir habilidades adecuadas para su edad. Existe gran variabilidad en la edad y en la adquisición o alcance de diferentes habilidades, esto es relevante porque nos hace referencia a la dificultad de establecer claramente un límite entre lo "normal" y lo "patológico". (Vericat, 2012)

En la Universidad Tecnológica del Suroeste de Guanajuato (UTSOE) ubicada en Valle de Santiago, Guanajuato, durante un año, se han evaluado a niños de 0 a 10 años para conocer acerca de su desarrollo psicomotor, planteándose la siguiente Hipótesis: El desarrollo psicomotor en los niños de 0 a 10 años en Valle de Santiago, Gto, es congruente con lo establecido en la escala de desarrollo infantil de Gesell. El DPM puede presentar variantes diversas como, retraso psicomotor, siendo este uno de los cuadros más frecuentemente detectados, también diferentes tipos de trastornos del desarrollo, así como problemas idiopáticos, todos estos solo son algunos ejemplos de este tipo de alteraciones.

\section{Signos de alarma}

A cualquier edad: macrocefalia (perímetro cefálico $[\mathrm{PC}]>+3$ desviaciones estándar [DS]), microcefalia ( $\mathrm{PC}<-2 \mathrm{DS}$ ), estancamiento del PC (tres o más meses sin aumentar), movimientos oculares anómalos excluido el estrabismo (movimientos erráticos, nistagmus, ojos en "sol poniente"), otros movimientos anómalos (actitudes distónicas, hiperextensión cefálica, movimientos cefálicos repetitivos de afirmación o negación), dismorfias obvias, arreflexia osteotendinosa generalizada (López, 2011). 2011):

A partir de edades concretas (Monge,

- Dos meses: irritabilidad permanente, sobresalto exagerado ante cualquier sonido inesperado, pulgar aducido (Más valor si es unilateral).

- Tres meses: asimetría de actividad en las manos. La lateralidad en el uso de las manos se obtiene alrededor de los dos años y no está firmemente establecida hasta los tres o cuatro años. Al valorar la manipulación, área motora fina, debemos comprobar que utiliza ambas manos.

- Cuatro meses: pasividad excesiva (pasa la mayor parte del tiempo durmiendo o sin apenas moverse y sin interesarse por su entorno), hipertonía de aductores (el ángulo de aductores de las extremidades inferiores no supera los 90).

- Seis meses: persistencia del reflejo de Moro (que se pierde habitualmente a los tres o cuatro meses), persistencia de la mirada a la mano.

- Ocho meses: patrón de conducta repetitiva más del 50\% del tiempo que pasa despierto (golpearse la cara o agitar las manos, movimientos de afirmación o negación cefálicos).

- Nueve meses: ausencia de desplazamiento autónomo más de dos metros (ya sea gateando, arrastrándose, volteándose, sentado $\mathrm{o}$, de cualquier forma).

- 12 meses: persistencia de llevarse todo a la boca, persistencia del babeo. 
- 16 meses: hiperactividad sin objeto (pasar constantemente de una actividad a otra, ser incapaz de mantener la atención o prestar interés por algo), persistencia de arrojar todo al suelo.

- 24 meses: estereotipias verbales o manuales (repetir constantemente los mismos sonidos o palabras), incapacidad de hacer juego simbólico (acunar o dar de comer a un muñeco, jugar a llamar por teléfono, jugar a indios y vaqueros).

\section{Signos de alarma detectados en UTSOE}

Se detectaron diversos signos de alarma dentro de la población de la Universidad, destacando: Aducción de pulgares $(0-5$ meses $): 15$ pacientes, atención deficiente (0-10 años): 13 pacientes, retraso en lenguaje y comunicación (0-10 años): 26 niños (Tabla 1).

\begin{tabular}{|c|c|c|}
\hline Signo de alarma & $\begin{array}{l}\text { Edad en } \\
\text { meses }\end{array}$ & $\begin{array}{l}\text { Número de } \\
\text { pacientes }\end{array}$ \\
\hline $\begin{array}{l}\text { Aducción de } \\
\text { pulgares }\end{array}$ & $0-5$ meses & 10 \\
\hline Irritabilidad & 0- 3 meses & 11 \\
\hline Marcha en punta & $0-6$ meses & 3 \\
\hline $\begin{array}{l}\text { Reflejo de } \\
\text { Hiperextensión }\end{array}$ & $0-9$ meses & 2 \\
\hline $\begin{array}{ll}\text { Retraso en el } \\
\text { lenguaje } \\
\text { comunicación }\end{array}$ & 0- 10 años & 26 \\
\hline $\begin{array}{l}\text { Atención } \\
\text { deficiente }\end{array}$ & 0-10 años & 3 \\
\hline Nistagmus & 0-10 años & 1 \\
\hline
\end{tabular}

Tabla 1 Signos de alarma en población pediátrica UTSOE Fuente: elaboración Propia

\section{Importancia del diagnóstico precoz de los problemas del desarrollo}

La detección precoz de trastornos del DPM ofrece la posibilidad de una intervención temprana y un tratamiento oportuno, lo cual permite corregir muchas de las alteraciones y atenuar otras (Schonhaut, 2008). De manera que se puede mejorar el pronóstico de los niños y la calidad de vida de sus familias. Se puede actuar a varios niveles:

- Prevención primaria: actuación sobre los niños de riesgo biológico y/o social mediante la disminución de dichos factores de riesgo y el incremento de los factores protectores.
- Prevención secundaria: actuación sobre los niños con retraso del desarrollo mejorando su funcionamiento y disminuyendo el grado de retraso.

- Tratamiento compensador: actuación sobre niños con discapacidades establecidas (síndrome de Down, parálisis cerebral infantil, etc.), no podemos cambiar la patología, pero podemos mejorar la calidad de vida del niño y su familia (Álvarez, 2009).

\section{Teoría del desarrollo de Arnold Gesell}

Arnold Gesell se dedicó a estudiar la interacción entre el desarrollo físico y mental, concluyendo que se produce una secuencia definida. Gesell sostenía que cada niño es único, con un código genético individual heredado con la capacidad de aprender. La teoría de Gesell es biológica considerando que el aprendizaje depende de la biología y fisiología del desarrollo y no a la inversa (Isaza, 2012).

Gesell considera que la organización del control de los actos motores sigue unas tendencias generales denominadas leyes $\mathbf{o}$ gradientes del desarrollo. (Justo, 2014).

- Ley o gradiente céfalo caudal: se refiere a que el desarrollo sigue un patrón regular conforme al cual las partes superiores del cuerpo comienzan a funcionar antes que las inferiores. Esto quiere decir que se controlan antes los movimientos de la cabeza que los de las piernas.

- Ley o gradiente general-especifico: los movimientos globales o generales aparecen antes que los más localizados, precisos y coordinados.

\section{Evaluación con escala de Gesell}

El test para la evaluación del desarrollo normal y anormal del niño de Arnold Gesell, nos permite evaluar al paciente pediátrico en edades clave, y en zonas de madurez. Las edades clave corresponden a tres períodos evolutivos, o zonas de madurez, que señalan la posición inicial habitual para el examen. 
Este test nos permite establecer las etapas de desarrollo, la descripción de cada edad, e identificar los modos de conducta observados, para interpretar su significación evolutiva en función de pautas normales. Se evaluaron 51 niños de 0 a 10 años de edad (ver Gráfico 1), de los cuales el sexo masculino es el más prevalente (ver Gráfico 2)

\section{Edad de los pacientes}

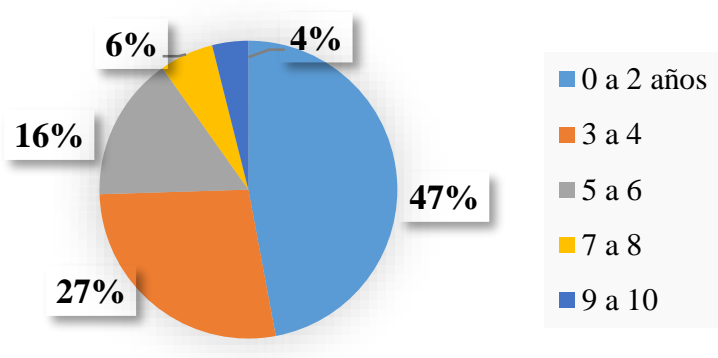

Gráfico 1 Edad de los pacientes evaluados con Gesell Fuente: elaboración propia

\section{Género más prevalente}

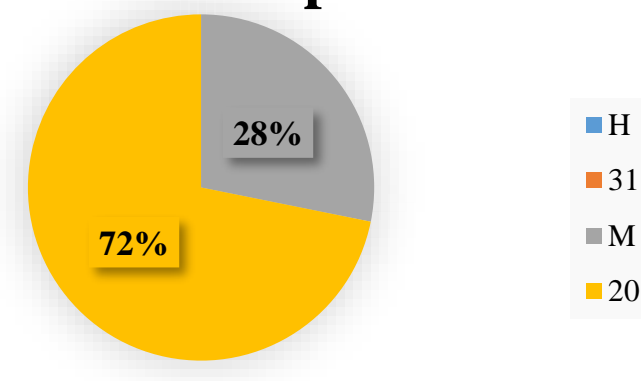

Gráfico 2 Género más prevalente de los pacientes evaluados con Gesell

Fuente: elaboración propia

Existen diversas alteraciones en el tono muscular que generan trastornos del movimiento $\mathrm{y}$ posturas anormales y prevalentes en el paciente pediátrico. En UTSOE se valoró el tono muscular de su población presentando con mayor frecuencia la hipertonía, hipotonía, espasticidad, mixto y normal (ver Gráfico 3).

\section{Tono muscular más común}

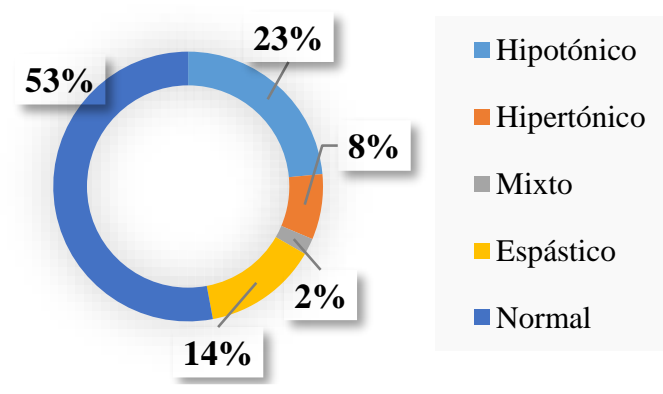

Gráfico 3 Valoración del tono muscular en pacientes pediátricos de UTSOE

Fuente: elaboración propia
Las áreas de desarrollo Psicomotor evaluadas corresponden al desarrollo global del niño, siendo estas 5 conductas: 1. Motor grueso que corresponde a movimientos que realiza el niño con músculos grandes (control de cuello, de tronco, gateo, bipedestación, giros, arrastre, marcha, salto, subir y bajar escaleras, patear una pelota, etc). (Gráfica 4)

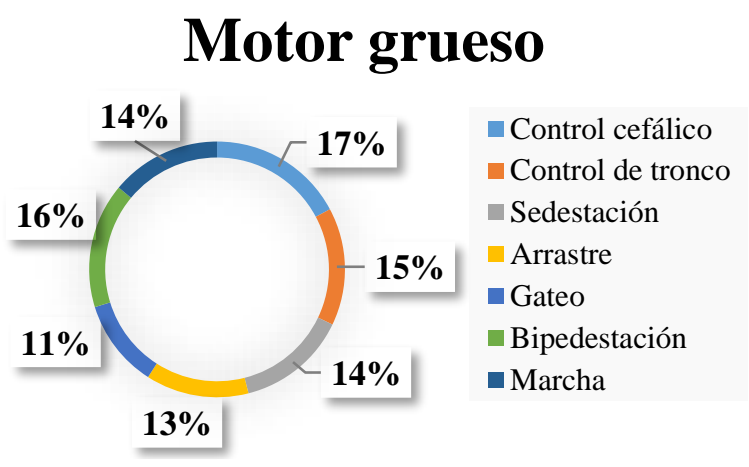

Gráfico 4 Valoración de conductas motrices gruesas en población UTSOE

Fuente: elaboración propia

Motor fino que corresponde a control de músculos pequeños de la mano y del pie como recortar, pintar, trazar líneas, introducir bolitas en una botella, cambiar las páginas de un libro, etc. (ver Tabla 2, ver Gráfico 5).

\begin{tabular}{|c|c|}
\hline $\begin{array}{l}\text { Motor fino } \\
\text { Actividad }\end{array}$ & Cumple \\
\hline Lleva las manos a la línea $n$ & $11 \%$ \\
\hline $\begin{array}{l}\text { Toma un objeto y lo transfiere entre sus } \\
\text { manos }\end{array}$ & $10 \%$ \\
\hline Desarrolla capacidad de agarre índice-pulgar & $9 \%$ \\
\hline Inserta objetos en un agujero grande & $12 \%$ \\
\hline Señala con el dedo índice & $10 \%$ \\
\hline Forma una torre de 2 cubos & $9 \%$ \\
\hline Introduce bolitas en una botella & $9 \%$ \\
\hline Da vuelta a las páginas de un libro & $10 \%$ \\
\hline Forma torre de 3 a 4 cubos & $10 \%$ \\
\hline Imita trazo vertical & $10 \%$ \\
\hline
\end{tabular}

Tabla 2 Valoración de conducta motriz fina en población UTSOE

Fuente: elaboración propia

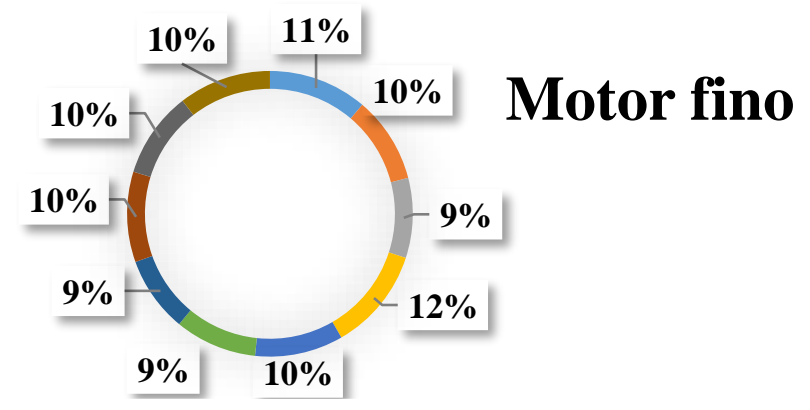

Gráfico 5 Resultados de conducta motriz fina en pacientes pediátricos de UTSOE

Fuente: elaboración propia

ALVAREZ-AVILA, Alejandra \& SASIA-ZAYAS, Karen. Reporte estadístico de pacientes pediátricos atendidos en la clínica de la Universidad Tecnológica del Suroeste de Guanajuato. Revista de Ciencias de la Salud. 2019 
3. Cognoscitivo se tranquiliza al oír la voz de la madre, respuesta vocal o gesticular ante un estímulo, señala partes del cuerpo cuando se le nombran, etc. (ver Tabla 3, ver Gráfico 6).

\begin{tabular}{|l|r|}
\multicolumn{1}{|c|}{$\begin{array}{c}\text { Cognoscitivo } \\
\text { Actividad }\end{array}$} & \multicolumn{1}{|c|}{ Cumple } \\
\hline Se tranquiliza al oír la voz de la madre & $9 \%$ \\
\hline Disminuye su actividad al escuchar un sonido & $11 \%$ \\
\hline Respuesta vocal ante un estímulo & $11 \%$ \\
\hline Responde con sonrisa social & $9 \%$ \\
\hline Examina los objetos antes de tomarlos & $12 \%$ \\
\hline $\begin{array}{l}\text { Se refiere a papá o mamá con cualquier } \\
\text { expresión }\end{array}$ & $10 \%$ \\
\hline Explora los objetos con la boca & $11 \%$ \\
\hline Descubre un objeto parcialmente escondido & $8 \%$ \\
\hline Señala 5 ilustraciones de 10 & $9 \%$ \\
\hline Señala partes del cuerpo cuando se le nombran & $10 \%$ \\
\hline
\end{tabular}

Tabla 3 Valoración de conducta cognoscitiva en población UTSOE

Fuente: elaboración propia

\section{Cognoscitivo}

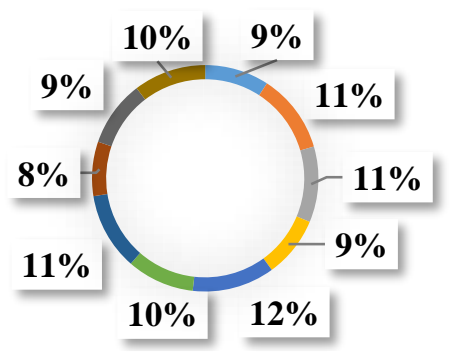

Gráfico 6 Resultados de conducta cognoscitiva en pacientes pediátricos de UTSOE

Fuente: elaboración propia

4. Lenguaje inicia gorjeo y cocleo, responde a sonrisa, usa fonemas, comprende palabras no y si, forma frases de 3 palabras, dice su nombre completo y enuncia preguntas, etc. (ver Tabla 4, ver Gráfico 7)

\begin{tabular}{|c|c|}
\hline $\begin{array}{l}\text { Lenguaje } \\
\text { Actividad }\end{array}$ & Cumple \\
\hline Realización de vocalización u a o & $7 \%$ \\
\hline Inicia gorjeo, arrullo y sonrisa & $8 \%$ \\
\hline Responde a su nombre & $10 \%$ \\
\hline Uso de fonemas bilabiales & $11 \%$ \\
\hline Comprensión aproximada de 17 palabras & $7 \%$ \\
\hline $\begin{array}{l}\text { Comprende la palabra NO acompañada del } \\
\text { gesto }\end{array}$ & $8 \%$ \\
\hline Emplea gestos de reconocimientos & $9 \%$ \\
\hline Cuenta con un vocabulario de 10 palabras & $12 \%$ \\
\hline Forma frases de 3 palabras & $9 \%$ \\
\hline Dice su nombre completo & $8 \%$ \\
\hline Enuncia preguntas & $11 \%$ \\
\hline
\end{tabular}

Tabla 4 Valoración de conducta de lenguaje en población UTSOE

Fuente: elaboración propia

\section{Lenguaje}

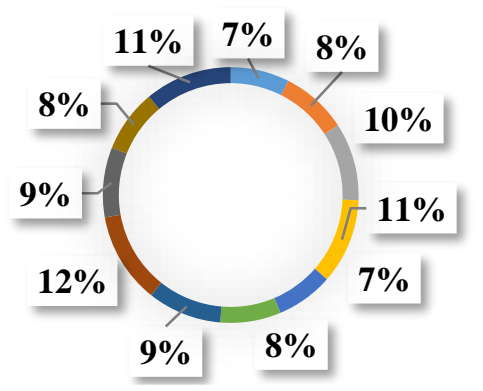

Gráfico 7 Resultados de conducta de lenguaje en pacientes pediátricos de UTSOE

Fuente: elaboración propia

5. Personal-social, sonríe en respuesta a una cara, no teme al peligro cuando explora, indica lo que desea, imita tareas domésticas, realiza actividades de la vida diaria por si solo como alimentarse, vestirse, ir al baño, peinarse y etc. (ver Tabla 5, ver Gráfico 8).

\begin{tabular}{|l|r|}
\hline \multicolumn{1}{|c|}{$\begin{array}{c}\text { Personal social } \\
\text { Actividad }\end{array}$} & Cumple \\
\hline Sonríe en respuesta a una cara & 49 \\
\hline $\begin{array}{l}\text { Sigue con la vista un objeto más allá de línea } \\
\text { media }\end{array}$ & 51 \\
\hline No teme al peligro cuando explora & 30 \\
\hline Indica lo que desea sin llorar & 38 \\
\hline Imita acciones (tareas domésticas) & 35 \\
\hline Ofrece un objeto con intención & 36 \\
\hline Se lava las manos & 29 \\
\hline Pide ayuda para expresar ideas & 38 \\
\hline Intenta contar experiencias & 41 \\
\hline Se pone prendas simples & 38 \\
\hline Come con cuchara & 34 \\
\hline
\end{tabular}

Tabla 5 Valoración de conducta personal social en población UTSOE

Fuente: elaboración propia

\section{Personal social}

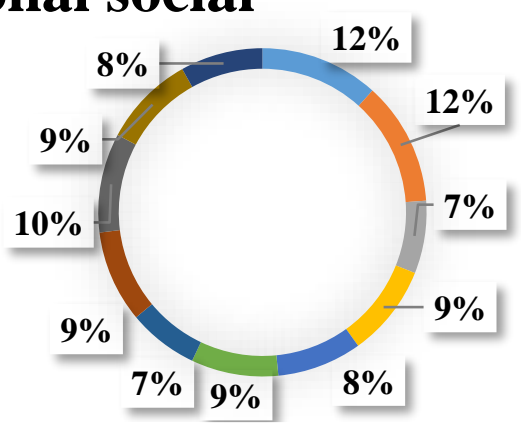

Gráfico 8 Resultados de conducta personal social en pacientes pediátricos de UTSOE

Fuente: elaboración propia 
Para poder realizar la valoración mediante Gesell, se genera de primera instancia, una historia clínica donde se toman en cuenta los factores de riesgo teniendo como resultado: 1. Prenatales (infecciones de vías urinarias como la más común con 22\%), 2. Perinatales (prematurez con $37 \%$ ), y postnatales (bajo peso al nacer con un $60 \%$, ver Gráfico 9).

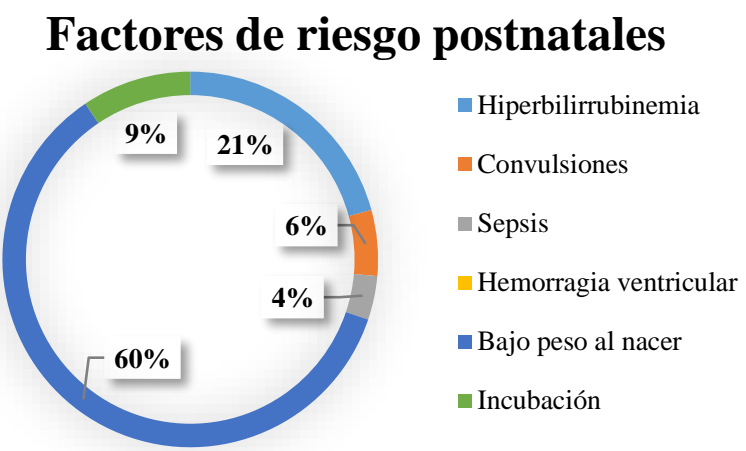

Gráfico 9 Factores más comunes de riesgo postnatal Fuente: elaboración propia

\section{Metodología a desarrollar}

Es un estudio observacional, descriptivo, de tipo transversal. Se evaluaron 51 pacientes, con la escala de Gesell, de entre 0-10 años.

La presente investigación se realizó en la Universidad Tecnológica del Suroeste de Guanajuato (UTSOE), durante el periodo de enero 2018 a Enero 2019.

Los criterios de inclusión son pacientes pediátricos de entre 0 a 10 años de edad que se pueden evaluar en áreas motor grueso, motor fino, lenguaje, adaptativo y personal social. Los criterios de exclusión son pacientes con discapacidad motriz, auditiva e intelectual, que tuvieran más de 10 años, que no se pudieran evaluar, y que tuvieran alguna enfermedad congénita

\section{Resultados}

Los resultados del artículo muestran que, de los 51 niños evaluados, el $29.41 \%$ presentan retraso en el área motor gruesa, el $25.44 \%$ tienen retraso en el área de lenguaje, siendo este uno de los aspectos posibles de un próximo estudio, el $15.68 \%$ retraso en conducta motriz fina, el 17.64 en conducta personal-social, y el 11.76 en conducta cognoscitiva (ver Tabla 6, Gráfico 9).
De los factores de riesgo, el que más se presenta es bajo peso al nacer, siendo este un factor de riesgo postnatal, y que con la correcta guía puede darse una solución para disminuir estos altos índices.

En las alteraciones del tono muscular, el tono tipo hipotónico es el más frecuente, se recomienda dar seguimiento, ya que esto puede generar alteraciones en el crecimiento del niño.

Se reciben con más frecuencia niños entre 3 a 4 años, por lo que se concluye que, en esta edad, los padres de familia comienzan a darse cuenta de retrasos en el desarrollo psicomotor, esto debido a que los infantes ingresan a educación preescolar, y los tutores tienen un referente, comparándolos con otros niños en las actividades que realizan.

\section{Agradecimiento}

Se agradece a la Universidad Tecnológica del Suroeste de Guanajuato, por las facilidades prestadas, permitir el uso de sus instalaciones, y fomentar la investigación.

\section{Conclusiones}

De acuerdo con la revisión que se tuvo durante este periodo, se denota que el aspecto motor grueso es el más afectado, seguido del área de lenguaje. Es necesario seguir con la investigación, para conocer los motivos de estos retrasos.

\section{Referencias}

Abdulqader, S. A., \& Saeed, B. A. (2019). Characteristics of patients attending the child and adolescent psychiatric outpatient clinic in Erbil city. PloS one, 14(2), e0209418.

Agustina Vericat; Alicia Bibiana. (2012). El desarrollo psicomotor y sus alteraciones: entre lo normal y lo patológico. De Instituto de Desarrollo e Investigaciones Pediátricas

Bahendeka, S., Mutungi, G., Tugumisirize, F., Kamugisha, A., Nyangabyaki, C., Wesonga, R., ... \& Were, T. P. (2019). Healthcare delivery for paediatric and adolescent diabetes in low resource settings: Type 1 diabetes clinics in Uganda. Global public health, 1-15. 
Eduardo Justo Martínez. (2014). Desarrollo psicomotor en educación infantil. Bases para la intervención en psicomotricidad. españa: Universidad Almería.

J. López Pisón, L. Monge Galindo. (2011). Evaluación y manejo del niño con retraso psicomotor. Trastornos generalizados del desarrollo. De Sección Neuropediatría. Hospital Universitario Miguel Servet.

Kam, K., Nettel-Aguirre, A., Mitchell, I., Johnson, D. W., \& Currie, G. (2019). A multidisciplinary clinic for pediatric patients with problematic severe asthma: Impact on clinical outcomes and healthcare utilization. Canadian Journal of Respiratory, Critical Care, and Sleep Medicine, 1-6.

Ko, C., Baird, M., Close, M., \& Cassas, K. J. (2019). The diagnostic accuracy of ultrasound in detecting distal radius fractures in a pediatric population. Clinical Journal of Sport Medicine, 29(5), 426-429.

Lomer, M. C., Cahill, O., Baschali, A., Sarathy, P. P., Sarantidou, M., Mantzaris, G. J., ... \& Gerasimidis, K. (2019). A multicentre Study of Nutrition Risk Assessment in Adult Patients with Inflammatory Bowel Disease Attending Outpatient Clinics. Annals of Nutrition and Metabolism, 74(1), 18-23

Luisa Schonhaut b. Jorge Álvarez 1. Patricia salinas a. (2008). El pediatra y la evaluación del desarrollo psicomotor. De revista chilena de pediatría.

M.J. Álvarez Gómeza, J. Soria Aznarb, J. Galbe Sánchez-Venturac. (2009). Importancia de la vigilancia del desarrollo psicomotor por el pediatra de Atención Primaria. De Rev Pediatr Aten Primaria.

Melissa Isaza Anyili Viviana López. (2012). propuesta didáctica según van hiele para el desarrollo de la noción de espacio en los niños y niñas de primero de primaria del liceo cuba de la ciudad de pereira-risaralda.. 2019, de universidad tecnológica de Pereira. 